\title{
Hadronic production of light color-triplet Higgs bosons: an alternative signature for GUT
}

\author{
Kingman Cheung ${ }^{1)}$ and Gi-Chol $\mathrm{Cho}^{2)}$ \\ 1 National Center for Theoretical Sciences, \\ National Tsing Hua University, Hsinchu, Taiwan, R.O.C. \\ 2 Department of Physics, Ochanomizu University, Tokyo 112-8610, Japan
}

(Dated: November 6, 2018)

\begin{abstract}
The conventional signature for grand unified theories (GUT) is the proton decay. Recently, some models in extra dimensions or with specific discrete symmetries, which aim at solving the doublet-triplet problem, allow the color-triplet in the $\mathrm{TeV}$ mass region by suppressing the Yukawa couplings of the triplets to matter fermions. We study the hadronic production and detection of these $\mathrm{TeV}$ colored Higgs bosons as an alternative signature for GUT, which would behave like massive stable charged particles in particle detectors producing a striking signature of a charged track in the central tracking system and being ionized in the outer muon chamber. We found that the LHC is sensitive to a colored Higgs boson up to about $1.5 \mathrm{TeV}$. If the color-triplets are stable in cosmological time scale, they may constitute an interesting fraction of the dark matter.
\end{abstract}

PACS numbers: 


\section{INTRODUCTION}

The doublet-triplet splitting problem is one of the most serious problems in supersymmetric (SUSY) grand unified theories (GUT) 1]. In SUSY-GUT, the weak-doublet Higgs fields which are responsible for the electroweak symmetry breaking belong to the $\mathbf{5}$ and $\overline{\mathbf{5}}$ representations of $\mathrm{SU}(5)$. They are composed of, in addition to the weak doublet, the color-triplet under the gauge group of the standard model (SM). After the SU(5) symmetry breaking, the weak doublets would still be massless while the color-triplets have to be decoupled from physics below the GUT scale, $M_{\mathrm{GUT}} \sim 10^{16} \mathrm{GeV}$, otherwise they may induce the proton decay in an experimentally unacceptable level through the Yukawa interactions to quarks or leptons in the SM. Most attempts to the doublet-triplet problem in the literatures have been focused on how to naturally explain the hierarchy between the weak doublet and the color-triplet Higgs boson masses after the GUT symmetry breaking [2].

An alternative approach to the doublet-triplet splitting problem, instead of requiring the triplet to have a mass of GUT scale, is to suppress the Yukawa couplings of the color-triplet Higgs fields to the SM quarks or leptons in order to preserve the proton longevity. Thus, no mass splitting between the weak-doublet and color-triplet Higgs fields is required. The natural scale of the color-triplet Higgs mass in this approach is the electroweak scale, say $O(100 \mathrm{GeV}) \sim O(1 \mathrm{TeV})$. Suppression of the Yukawa couplings of the triplet is possible via the following mechanisms.

- The SM fermion masses are generated from a higher dimensional operators involving the GUT Higgs field in the adjoint representation, the VEV of which can be arranged such that the Yukawa couplings of the doublets get the usual values while the Yukawa couplings of the triplets are zero $[3]$.

- The overlap of the wave-functions of the color-triplet Higgs fields with the SM fermions in extra dimensions are sufficiently small [4].

- Another type of models is based on orbifolding in AdS space. By assigning different spatial parities to various components of the Higgs multiplet, the wave-function of the color-triplet Higgs fields are zero at the Planck brane, on which the matter fermions

reside [5]. Thus, the excessive proton decay via the the colored Higgs boson is highly suppressed. In addition, the mass of the color-triplet fields is given by the warp factor 
of the AdS and naturally of a TeV scale.

We shall describe these models in some details in the next section.

The generic feature of these models to the doublet-triplet problem is the presence of colored Higgs bosons in TeV scale. In fact, this is an alternative novel signature to the conventional signature of proton decay, and can be tested in the upcoming LHC. It is then worthwhile to investigate the phenomenology of the TeV colored Higgs bosons in collider experiments at high energy frontier. In hadronic collisions, the colored Higgs bosons are produced via the glue-glue fusion and the $s$-channel $q \bar{q}$ annihilation. On the other hand, it is also possible to produce pairs of colored Higgs bosons at $\mathrm{TeV} e^{+} e^{-}$linear colliders and $\gamma \gamma$ colliders via the $\mathrm{U}(1)_{Y}$ gauge boson exchange, but the production rates are suppressed relative to hadronic production because of the small $\mathrm{U}(1)_{Y}$ coupling.

In this paper, we calculate the production and describe the detection of the $\mathrm{TeV}$ colored Higgs bosons in hadron colliders. As we already mentioned, the colored Higgs bosons do not have sizable Yukawa couplings to the SM fermions in order to suppress the fast proton decay. Thus, the only allowed production channels of the colored Higgs bosons in hadronic collisions are via the $\mathrm{SU}(3)_{C}$ invariant interactions. The colored Higgs bosons have a distinctive feature that gives rise to a novel signature like a "heavy muon". Since they have no Yukawa couplings and can only pair-wise couple to gluons, the colored Higgs bosons cannot decay into the SM particles directly. They may be able to decay into a gluino and a colored higgsino through the gaugino-matter interactions only if it is kinematically allowed, though. However, in our analysis, we assume that the colored Higgs boson has a mass less than the sum of gluino and colored higgsino masses so that such a decay channel is forbidden. This is a reasonable assumption because we expect the colored higgsino to have a mass scale as heavy as the colored Higgs boson. Therefore, the colored Higgs bosons produced in hadron colliders are stable, and will be hadronized into color-neutral massive particles by combining with gluons or light quarks. Statistically, we also assume that half of the time the colored Higgs boson will be an electrically-charged particle after hadronization. ${ }^{1}$ This is the reason why it will behave like a "heavy muon" in the detector. The novel signature will then be an

\footnotetext{
${ }^{1}$ The probability of hadronizing into a charged particle depends on the spectrum of the bound states of the colored Higgs boson with the light degrees of freedom. However, there does not exist any realistic calculations of the spectrum, and so we take the probability to be $1 / 2$.
} 
observable charged track in the central tracking chamber and/or the silicon vertex detector and a penetration to the outer muon chamber - heavy muon. Taking into account of all these properties, we study the discovery potential of the colored Higgs bosons in the upcoming hadron colliders, the LHC and the VLHC.

This paper is organized as follows. In the next section, we briefly describe the three models mentioned above that allow light color-triplet Higgs fields. In Sec. III, we review the interactions of colored Higgs bosons and briefly discuss the indirect constraints. In Sec. IV we discuss the conditions to detect the colored Higgs bosons. The hadronic production of the colored Higgs bosons and their detectability in collider experiments will be discussed in Sec. $\square$ The last section will be devoted to a summary and discussion.

\section{MODELS OF TEV COLOR-TRIPLET}

In this section, we highlight on three models that allow light $(\mathrm{TeV})$ color-triplet Higgs bosons by Dvali [3], by Haba and Maru [4], and by Goldberger, Nomura and Smith [5].

In supersymmetric GUT models, the Higgs doublets that give Yukawa couplings to fermions are accompanied by the color-triplet in the same multiplet. The color-triplet couples to fermions with the same Yukawa couplings as the doublet before the GUT symmetry is broken. That is why the color-triplet has to be extremely heavy in order to avoid the proton decay. However, the proton decay problem can also be solved if the Yukawa couplings of the color-triplet are suppressed relative to the doublet by a ratio $M_{W} / M_{\mathrm{GUT}}$, and thus the color-triplet needs not to be heavy and can be as light as the doublet. Imposing a specific discrete symmetry to forbid the lowest order Yukawa term $16^{\alpha} 16^{\beta} 10$, the Yukawa couplings of the doublet have to be generated via higher dimensional terms. Dvali [3] constructed a higher dimensional $\mathrm{SO}(10)$ invariant operator

$$
\frac{Y_{\alpha \beta}}{M} 10_{i} 45_{i k} 16^{\alpha} \gamma_{k} 16^{\beta}
$$

where $M \sim M_{\mathrm{GUT}}$, the matter fermions reside in the $\mathbf{1 6}, \alpha, \beta$ are family indices, $\mathbf{1 0}_{i}$ consists of color-triplets $(i=1, \ldots, 6)$ and doublets $(i=7, \ldots, 10)$, and $\mathbf{4 5}$ is the GUT Higgs in the adjoint representation of $\mathrm{SO}(10)$. This term can arise from tree-level exchanges of heavier states at $M_{\text {GUT }}$. Taking a VEV for the $\mathbf{4 5}$ as

$$
\left\langle\mathbf{4} \mathbf{5}_{i k}\right\rangle=\operatorname{diag}(0,0,0, A, A) \otimes \epsilon
$$


where $A \sim M_{\mathrm{GUT}}$ and $\epsilon$ is the $2 \times 2$ antisymmetric matrix, and substituting into the above operator, it is easy to see that the doublet gets a Yukawa coupling $Y_{\alpha \beta} A / M$ while the colortriplet has zero Yukawa couplings. In this case, the color-triplet would not cause any proton decay and thus can be as light as the doublet. Of course, the usual $16^{\alpha} \mathbf{1 6}^{\beta} \mathbf{1 0}$ Yukawa term is absent because of a specific discrete symmetry. Dvali was using a $Z_{2} \times Z_{3}[3]$.

Another interesting solution to the doublet-triplet problem was recently proposed by Haba and Maru 4]. Although it is of similar spirit, the setup is however entirely different. Basically, in the setup of extra dimensions the proton stability is maintained by suppressing the Yukawa couplings of the color-triplet to matter fermions through the small overlap of wave-functions in the extra dimensions. They started with a $\mathrm{SU}(5)$ model in 5 dimensions with the Higgs doublets and triplets in $\mathbf{5}$ and $\overline{\mathbf{5}}$, the usual SU(5) GUT adjoint Higgs field being assumed to localize on the brane at $y=0$ ( $y$ is the coordinate in the extra dimension), and a pair of bulk fields in 24. Through interactions with the bulk fields (very similar to the idea of domain-wall fermions), the zeroth mode of the Higgs doublets are localized in a position close to the brane at $y=0$, where the matter fermions are confined, while the color-triplet Higgs fields are localized at a further distance from the brane at $y=0$. By varying the distance between the doublets and triplets, the Yukawa couplings of the triplets can be sufficiently suppressed to avoid the proton decay while the doublets can generate the usual Yukawa couplings to the matter fermions. Therefore, the color-triplets need not be heavy and can be as light as the weak doublets.

The third type of models is based on orbifolding. By assignment of different spatial parities (or boundary conditions) to various components of a multiplet, the component fields can have very different properties at the fixed points. Thus, it is possible to break a symmetry or to achieve the doublet-triplet splitting by the boundary conditions. In the model by Goldberger et al. [5], they started from the Randall-Sundrum scenario [6] : a slice of AdS space with two branes (the Planck brane and the $\mathrm{TeV}$ brane) at both ends. The extra dimension is compactified on an $S^{1} / Z_{2}$ orbifold. The hierarchy of scales is generated by the AdS warp factor $k$, which is of order of the five-dimensional Planck scale $M_{5}$, such that the $4 \mathrm{D}$ Planck scale is given by $M_{\mathrm{Pl}}^{2} \sim M_{5}^{3} / k$. The fundamental scale on the Planck brane is $M_{\mathrm{Pl}}$ while the fundamental scale on the $\mathrm{TeV}$ brane is rescaled to $\mathrm{TeV}$ by the warp factor: $T \equiv k e^{-\pi k R}$, where $R$ is the size of the extra dimension. The model is a $5 \mathrm{D}$ supersymmetric $\mathrm{SU}(5)$ gauge theory compactified on the orbifold $S^{1} / Z_{2}$ in the AdS space. 
The boundary conditions break the SU(5) symmetry and provide a natural mechanism for the Higgs doublet-triplet splitting and suppress the proton decay. The Planck brane respects the SM gauge symmetry while the TeV brane respects the SU(5) symmetry. The matter fermions reside on the Planck brane. By the boundary conditions the wave-function of the color-triplet Higgs fields are automatically zero at the Planck brane, on which the matter fermions reside, while the doublet Higgs fields are nonzero at the Planck brane and give Yukawa couplings to the matter fermions. Thus, the excessive proton decay via the colortriplet Higgs fields is highly suppressed, and the doublet-triplet splitting is therefore natural by the boundary conditions. The mass of the color-triplet fields (and the XY guage bosons) is given by the warp factor and is of a TeV scale, the same as the KK states of other fields in the setup. This model is very similar, in spirit, to the second model in suppressing the proton decay by a very small or zero overlap of the wave-functions, but the mechanism is more natural in this model. In addition, the mass of the color-triplet is also naturally given by the warp factor in the $\mathrm{TeV}$ scale.

\section{REVIEW OF LIGHT COLORED HIGGS BOSON}

The two Higgs fields in the SUSY-GUT, $H(\mathbf{5})$ and $H(\overline{\mathbf{5}})$, are composed of the weak doublet and the color triplet as follows:

$$
\begin{aligned}
& H(\mathbf{5})=\left(H_{C}, H_{u}\right), \\
& H(\overline{\mathbf{5}})=\left(\bar{H}_{C}, H_{d}\right),
\end{aligned}
$$

where the weak doublets $H_{u}$ and $H_{d}$ are responsible for the up- and the down-type quark (lepton) masses, respectively. The color-triplets $H_{C}$ and $\bar{H}_{C}$ could be as light as $O(\mathrm{TeV})$ via the mechanisms that we have described above. The price for the TeV colored Higgs bosons is the absence of the Yukawa interactions of $H_{C}$ and $\bar{H}_{C}$ to the SM fermions. The colored Higgs boson couples to the gluon $A_{\mu}^{a}$ through the following interactions:

$$
\mathcal{L}=-i g_{s} H_{C}^{*} \stackrel{\leftrightarrow}{\partial}_{\mu} H_{C} T^{a} A^{a \mu}+g_{s}^{2} T^{a} T^{b} H_{C}^{*} H_{C} A_{\mu}^{a} A^{b \mu}
$$

where $T^{a}$ is the generator of $\mathrm{SU}(3)$, and $A \overleftrightarrow{\partial}_{\mu} B \equiv A\left(\partial_{\mu} B\right)-\left(\partial_{\mu} A\right) B$. The interactions for $\bar{H}_{C}$ is the same as $H_{C}$ in Eq. (3). The production of the colored Higgs bosons in the lowest order is via the $s$-channel $q \bar{q}$ annihilation and the glue-glue fusion, shown by the Feynman diagrams in Figs. 1 and 2 . 


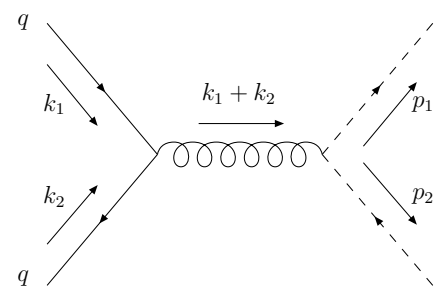

FIG. 1: Feynman diagram for $q \bar{q} \rightarrow H_{C} H_{C}^{*}$.
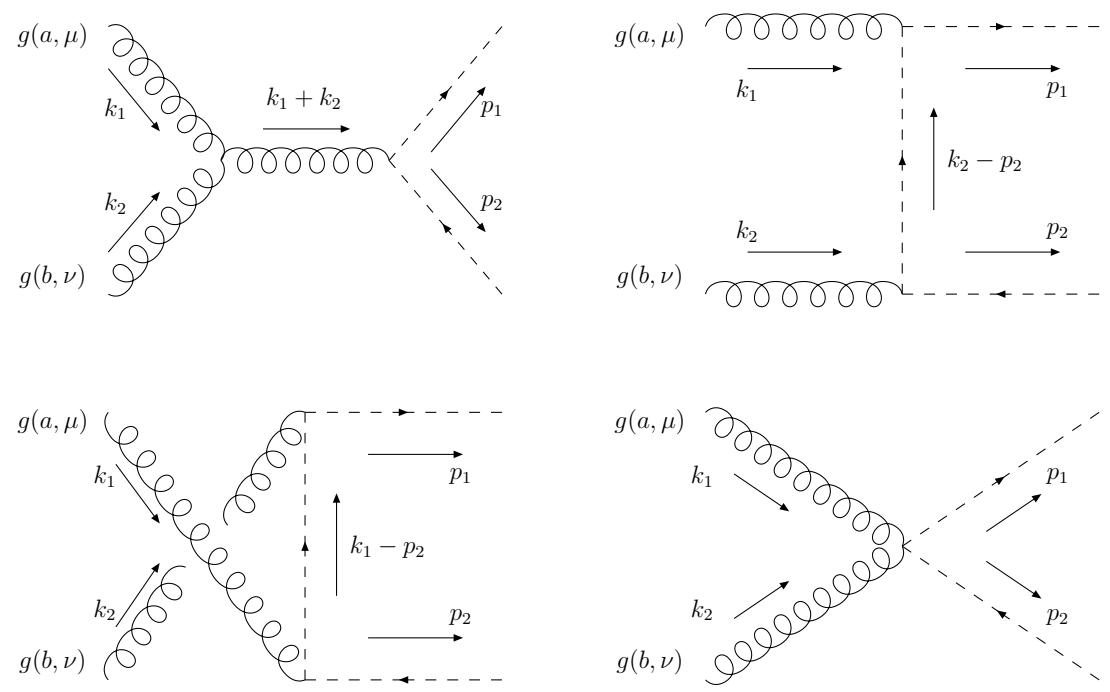

FIG. 2: Feynman diagrams for $g g \rightarrow H_{C} H_{C}^{*}$.

In addition to Eq. (3) the colored Higgs boson also interacts with the colored higgsino $\widetilde{h}_{C}$ and the gluino $\widetilde{g}$ :

$$
\mathcal{L}=-\sqrt{2} g_{s}\left(H_{C}^{*} \overline{\widetilde{g}^{a}} T^{a} \widetilde{h}_{C}+\overline{\widetilde{h}_{C}} \widetilde{g}^{a} T^{a} H_{C}\right)
$$

However, as we already mentioned, we have assumed that the colored Higgs bosons do not decay into colored higgsinos and gluinos because it is not allowed kinematically.

Is there any indirect constraints on the colored Higgs bosons from the high-energy experiments? Let us consider the $Z$-pole experiments at LEP1 and SLC where the radiative corrections to the gauge boson propagators and the $Z \rightarrow f \bar{f}$ ( $f$ denotes quarks or leptons) vertices are severely constrained. The contributions to the gauge boson propagators are summarized by the $S, T, U$ parameters [7]. It is well known that the $\mathrm{SU}(2)_{L}$ singlet scalars do not contribute to the $S, T, U$ parameters [8] such that the colored Higgs boson is free from 
the constraints. Since the Yukawa interactions of $H_{C}$ and $\bar{H}_{C}$ to the quarks and leptons are highly suppressed, they do not contribute to the $Z \rightarrow f \bar{f}$ processes. By the same reason, there is no constraint on the colored Higgs bosons from flavor physics experiments. We, therefore, conclude that no indirect constraints are implied for the colored Higgs mass from current experiments.

We comment on the gauge coupling unification before closing this section. It is well known that the success of gauge coupling unification in the minimal supersymmetric standard model (MSSM) could be preserved if complete multiplets of SU(5) (e.g., 5 or $\mathbf{1 0} \cdots)$ are added to the spectrum of MSSM. When $H_{C}$ and $\bar{H}_{C}$ are in TeV scale, there must be a vector pair of weak doublets in the same scale to form the $\mathbf{5}$ and $\overline{\mathbf{5}}$ multiplets so that the gauge coupling unification is unaltered. The origin of the weak vector-like pair is discussed in both Refs. [3] and [4].

\section{DETECTION OF MASSIVE CHARGED PARTICLES}

The colored Higgs boson will hadronize into a massive stable particle, which is electrically either neutral or charged. Both the neutral and charged particles will undergo hadronic interactions with detector materials while the charged particle will also undergo ionization, through which the particle loses its kinetic energy (K.E.). Such a massive stable particle will have a high transverse momentum and a small velocity (or $\beta=v / c$ ). Furthermore, if it is charged it will penetrate detector materials like a muon.

First, we discuss the hadronic energy loss of a massive stable particle in the detector (both neutral and charged should have similar behavior.) Although it is strongly interacting, it penetrates the material with a very small loss of energy. This is because the energy loss in hadronic elastic scattering is negligible because of the small momentum transfer. In addition, the energy loss in elastic or inelastic scattering with nucleons is also small because of the huge mass difference between the massive particle $(\mathrm{TeV})$ and the nucleon. Thus, the energy loss via hadronic collisions does not lead to detection of the massive particle. If the colored

Higgs hadronizes into a neutral particle, it will escape the detector and undetected. The detection of colored Higgs bosons has to rely upon the charged state, which will be described next.

The energy loss $d E / d x$ due to ionization with the detector material is very standard [9]. 
Essentially, the penetrating particle loses energy by exciting the electrons of the material. The basic formula can be found in Eq. (26.1) in Particle Data Book (PDB) 9]. We shall ignore the small correction from the density effect. Ionization energy loss $d E / d x$ is a function of $\beta \gamma$ and the charge $Q$ of the penetrating particle. The dependence on the mass $M$ of the penetrating particle comes in through $\beta \gamma$ for a large mass $M$ and small $\gamma[9]$. In other words, $d E / d x$ is the same for different masses if the $\beta \gamma$ and $\beta$ values of these particles are the same. In Fig. 3. we show $d E / d x$ for a wide mass range $10^{-3}-10^{4} \mathrm{GeV}$ as a function of $\beta \gamma$. For the range of $\beta \gamma$ between 0.1 and 1 that we are interested in, $d E / d x$ has almost no explicit dependence on the mass $M$ of the penetrating particle. Therefore, when $d E / d x$ is measured in an experiment, the $\beta \gamma$ can be deduced, which then gives the mass of the particle if the momentum $p$ is also measured. Hence, $d E / d x$ is a good tool for particle identification for massive stable charged particles.

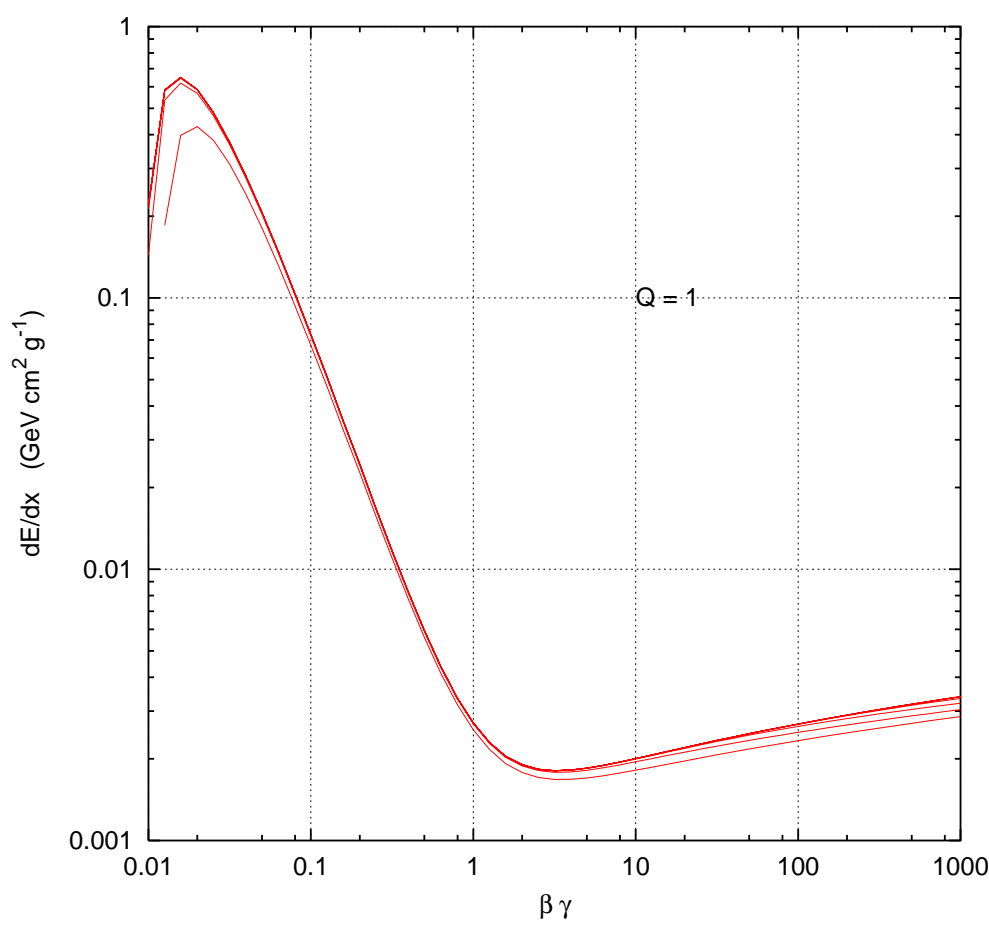

FIG. 3: $d E / d x$ calculated using the formula in PDB for a range of $M=10^{-3}-10^{4} \mathrm{GeV} \cdot M=10^{-3}$ $\mathrm{GeV}$ is for the bottom of the lines and goes up to $M=10^{4} \mathrm{GeV}$ for the top of the lines. It is for $\mathrm{Si}$ (we used a value of $I=2 \mathrm{eV}$ for illustration.)

In fact, the CDF Collaboration did a few searches for massive stable charged particles 10, 11, 12]. The CDF analyses require that the particle produces a track in the central tracking chamber and/or the silicon vertex detector, and at the same time penetrates to 
the outer muon chamber. The CDF detector has a central tracking chamber and a silicon vertex detector (the central tracking chamber has a slightly better resolution in this regard), which can measure the energy loss $(d E / d x)$ of a particle via ionization, especially at low $\beta \gamma<0.85(\beta<0.65)$ where $d E / d x \sim 1 / \beta^{2}$. Once the $d E / d x$ is measured, the mass $M$ of the particle can be determined if the momentum $p$ is measured simultaneously. Furthermore, the particle is required to penetrate through the detector material and make it to the outer muon chamber, provided that it has an initial $\beta>0.25-0.45$ depending on the mass of the particle [10]. Therefore, the CDF requirement on $\beta$ or $\beta \gamma$ is

$$
0.25-0.45 \lesssim \beta<0.65 \Leftrightarrow 0.26-0.50 \lesssim \beta \gamma<0.86
$$

The lower limit is to make sure that the penetrating particle can make it to the outer muon chamber while the upper limit makes sure that the ionization loss in the tracking chamber is sufficient for a detection. We shall employ a similar requirement in our analysis that we shall illustrate next.

Here we verify that the lower limit on $\beta \gamma$ is valid for a $1 \mathrm{TeV}$ charged particle with $Q=1$. We use the $d E / d x$ formula in PDB for Si (we choose the value $I=2 \mathrm{eV}$ for the illustration purpose.) We calculate the minimum value of $\beta \gamma$ for a singly-charged particle of mass $M$ to penetrate a silicon of a $5 \mathrm{~m}$ thickness (it is roughly equivalent to a $1.5 \mathrm{~m}$ of $\mathrm{Fe}$ because the density of $\mathrm{Fe}$ is 3.4 times of that of Si.) The calculation procedures are rather straightforward and described as follows. (i) The particle of mass $M$ starts with an initial $\beta \gamma$. (ii) Calculate the value of $d E / d x$ and let the particle penetrate for a step of $1 \mathrm{~cm}$. (iii) Evaluate the remaining energy and calculate the corresponding $\beta \gamma$, which is then input to calculate the next $d E / d x$ in the next step $(1 \mathrm{~cm})$. (iv) Repeat until the $\beta \gamma$ goes to zero or the particle penetrates a distance of $5 \mathrm{~m}$. If $\beta \gamma$ goes to zero before reaching $5 \mathrm{~m}$, the particle does not have a complete penetration, else the particle completes the penetration. We show the minimum $\beta \gamma$ values for a complete penetration of a $5 \mathrm{~m}$ Si or equivalent vs the mass $M$ of the penetrating particle in Fig. 4. From the figure, the minimun $\beta \gamma$ for a particle of mass between $50-500 \mathrm{GeV}$ is about $0.18-0.44$, which is consistent with the CDF requirement in Eq. (5). For a $1 \mathrm{TeV}$ particle, the $(\beta \gamma)_{\min }$ is about 0.14 . Therefore, in our analysis we shall choose a conservative value of $(\beta \gamma)_{\min }=0.25$, and our final cut on $\beta \gamma$ is

$$
0.25 \leq \beta \gamma \leq 0.85
$$


Here we have chosen the upper limit very close to what CDF used [10, 11, 12], because $d E / d x$ is almost independent of the mass of the penetrating particle in the range $0.1<\beta \gamma<1$ (see Fig. 3). Essentially, the upper limit ensures that the particle deposits a sufficient amount of energy in the central tracking system and the muon system for detection, beyond which the $d E / d x$ may be too small for detection.

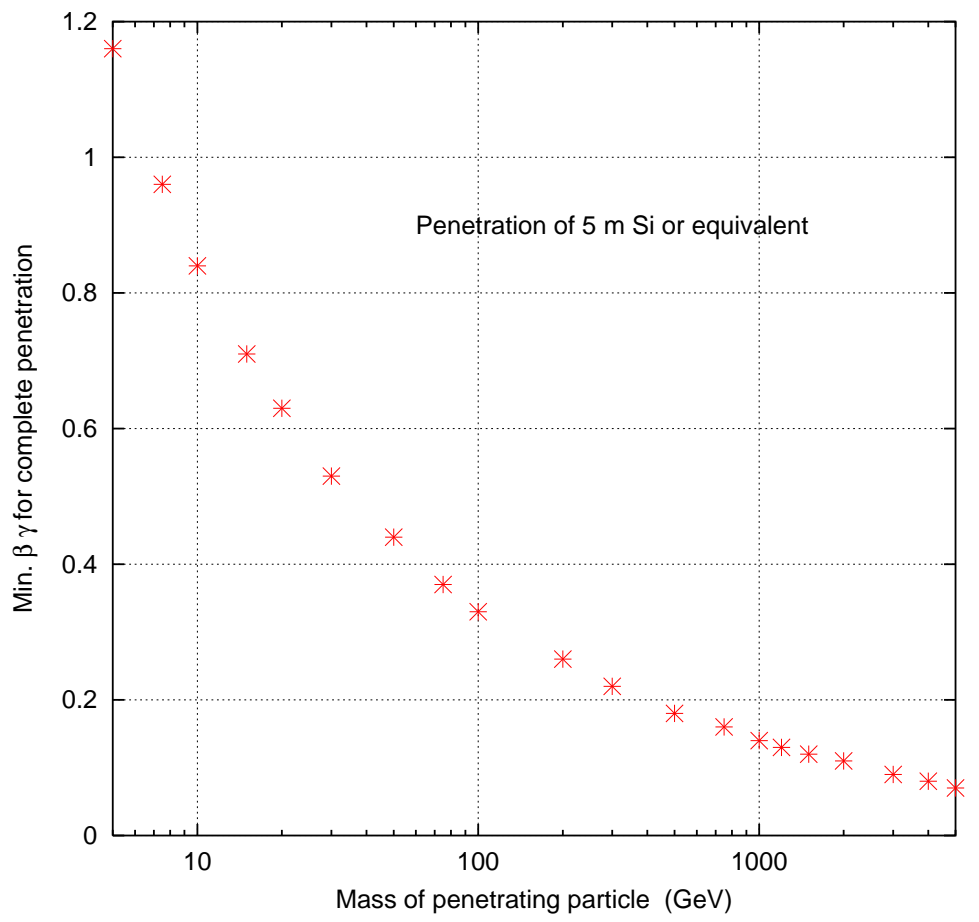

FIG. 4: Minimum $\beta \gamma$ values for a complete penetration of a $5 \mathrm{~m} \mathrm{Si}$ or equivalent vs the mass $M$ of the penetrating particle.

Since the lower cut on $\beta \gamma=p / M$ is 0.25 , the momentum cut is $250 \mathrm{GeV}$ for a $1 \mathrm{TeV}$ particle. Such a cut on momentum already makes it background free from $\mu^{ \pm}, \pi^{ \pm}, K^{ \pm}$ together with the cut on $\beta \gamma$. Another configuration cut due to the detector (both CMS and Atlas) is

$$
|\eta|<2.5
$$

We also assume an efficiency of $80 \%$ for each massive stable charged particle to be detected by the central tracking system and the outer muon system. This efficiency is in addition to the cuts on $\eta$ and $\beta \gamma$.

There were also some theoretical studies on search for massive stable charged particles, such as a light gluino as the LSP 13] and a scalar tau in the context of gauge mediated supersymmetry breaking models [14]. 


\section{PRODUCTION AT HADRONIC SUPERCOLLIDERS}

Pairs of colored Higgs bosons can be produced via $q \bar{q}$ and $g g$ fusion. The color- and spin-averaged amplitude squared are given by

$$
\begin{aligned}
\bar{\sum}\left|M\left(g g \rightarrow H_{C} H_{C}^{*}\right)\right|^{2} & =\frac{g_{s}^{4}}{128}\left[24\left(1-\frac{2 \hat{t}_{m} \hat{u}_{m}}{\hat{s}^{2}}\right)-\frac{8}{3}\right]\left[1-\frac{2 \hat{s} m_{H_{C}}^{2}}{\hat{u}_{m} \hat{t}_{m}}\left(1-\frac{\hat{s} m_{H_{C}}^{2}}{\hat{u}_{m} \hat{t}_{m}}\right)\right] \\
\bar{\sum}\left|M\left(q \bar{q} \rightarrow H_{C} H_{C}^{*}\right)\right|^{2} & =\frac{4 g_{s}^{4}}{9} \frac{\hat{t} \hat{u}-m_{H_{C}}^{4}}{\hat{s}^{2}} .
\end{aligned}
$$

In the above equations, we have defined

$$
\begin{aligned}
\hat{t}_{m} & \equiv \hat{t}-m_{H_{C}}^{2}=-\frac{\hat{s}}{2}(1-\beta \cos \theta) \\
\hat{u}_{m} & \equiv \hat{u}-m_{H_{C}}^{2}=-\frac{\hat{s}}{2}(1+\beta \cos \theta),
\end{aligned}
$$

where $\theta$ is the scattering angle and $\beta=\sqrt{1-4 m_{H_{C}}^{2} / \hat{s}}$ is the velocity of the outgoing Higgs bosons in the center-of-mass frame of the incoming partons.

The parton-level differential cross sections are then given by:

$$
\begin{aligned}
\frac{d \hat{\sigma}}{d \cos \theta}\left(g g \rightarrow H_{C} H_{C}^{*}\right) & =\frac{\pi \alpha_{s}^{2} \beta}{256 \hat{s}}\left[24\left(1-\frac{2 \hat{t}_{m} \hat{u}_{m}}{\hat{s}^{2}}\right)-\frac{8}{3}\right]\left[1-\frac{2 \hat{s} m_{H_{C}}^{2}}{\hat{u}_{m} \hat{t}_{m}}\left(1-\frac{\hat{s} m_{H_{C}}^{2}}{\hat{u}_{m} \hat{t}_{m}}\right)\right](12) \\
\frac{d \hat{\sigma}}{d \cos \theta}\left(q \bar{q} \rightarrow H_{C} H_{C}^{*}\right) & =\frac{2 \pi \alpha_{s}^{2} \beta}{9 \hat{s}} \frac{\hat{t} \hat{u}-m_{H_{C}}^{4}}{\hat{s}^{2}}
\end{aligned}
$$

After integrating over the angle $\theta$, the parton-level cross sections are given by

$$
\begin{aligned}
\hat{\sigma}\left(g g \rightarrow H_{C} H_{C}^{*}\right) & =\frac{\pi \alpha_{s}^{2}}{\hat{s}}\left(\beta \frac{5 \hat{s}+62 m_{H_{C}}^{2}}{48 \hat{s}}+\frac{m_{H_{C}}^{2}}{6 \hat{s}} \frac{4 \hat{s}+m_{H_{C}}^{2}}{\hat{s}} \log \frac{1-\beta}{1+\beta}\right), \\
\hat{\sigma}\left(q \bar{q} \rightarrow H_{C} H_{C}^{*}\right) & =\frac{\pi \alpha_{s}^{2} \beta}{\hat{s}}\left(\frac{2}{27}-\frac{8}{27} \frac{m_{H_{C}}^{2}}{\hat{s}}\right) .
\end{aligned}
$$

The above results agree with the cross sections of squark-pair production in Ref. [15] if only the $s$-channel process is taken into account in the $q \bar{q}$ annihilation. Note that the expressions for the production cross sections of the $\bar{H}_{C} \bar{H}_{C}^{*}$ pair are the same as the $H_{C} H_{C}^{*}$ pair. If the mass of $\bar{H}_{C}$ is the same as $H_{C}$, the sum of the cross sections would be doubled. In the minimal SUSY SU(5), they have exactly the same mass. Even beyond the minimal model, since there is no particular reason why their masses should be very different, we simply take them to be equal and the results present in the following take into account both $H_{C}$ and $\bar{H}_{C}$. 
In the calculation, we employ the parton distribution function of CTEQ v.5 (set L) 16] and the one-loop renormalized running strong coupling constant with $\alpha_{s}\left(M_{Z}\right)=0.119$. The total cross section at a center-of-mass energy $\sqrt{s}$ is obtained by convoluting the partonic cross sections in Eqs. (14) and (15) with the parton distribution functions:

$$
\sigma(s)=\int_{4 m_{H_{C}}^{2} / s}^{1} d \tau \int_{\tau}^{1} \frac{d x}{x} f(\tau / x) f(x) \hat{\sigma}(\hat{s}),
$$

where $\hat{s}=\tau s$ is square of the center-of-mass energy of the parton-parton scattering.

We show in Fig. [5 the production cross sections vs the mass $m_{H_{C}}$ of the colored Higgs boson at the $2 \mathrm{TeV}$ Tevatron ( $p \bar{p}$ collisions), at the LHC ( $p p$ collisions), and in $p p$ collisions at $\sqrt{s}=50,200 \mathrm{TeV}$. The acceptance cut on the pseudo-rapidity in Eq. (17) has been applied. As explained above this acceptance is due to the coverage of the central tracking chamber. We have shown the cross sections at 50 and $200 \mathrm{TeV}$ center-of-mass energies, which is, respectively, the lower and upper energy range of the VLHC. The VLHC (very large hadron collider) is another $p p$ accelerator under discussions 17] in the Snowmass 2001 [18]. The preliminary plan is to have an initial stage of about $40-60 \mathrm{TeV}$ center-of-mass energy, and later an increase up to $200 \mathrm{TeV}$. The targeted luminosity is $(1-2) \times 10^{34} \mathrm{~cm}^{-2} \mathrm{~s}^{-1}$.

The next important distribution in our analysis is the $\beta \gamma=p / M$ distribution. We show the normalized differential cross section $1 / \sigma d \sigma / d(\beta \gamma)$ vs $\beta \gamma$ for $m_{H_{C}}=0.5,1,1.5 \mathrm{TeV}$ at the LHC in Fig. 6. The majority of the cross section is below $\beta \gamma \simeq 1.2$ as the heavy colored Higgs bosons are produced not too far away from the threshold. Thus, the velocity of the Higgs bosons is not too large. It is obvious that the heavier the boson, the smaller is the average velocity $\beta$. We expect that when we apply the selection cut of Eq. (6) on the colored Higgs bosons, a majority of the cross section remains. We have verified that if we require at least one of the colored Higgs bosons for $m_{H_{C}}=1 \mathrm{TeV}$ satisfying the $\beta \gamma$ cut of Eq. (6), about $60 \%$ of the cross section remains.

Since we have assumed the detection efficiency of a track is $80 \%$ in addition to the acceptance cuts on $\eta$ and $\beta \gamma$, the combined efficiency to see two tracks would be $(0.8)^{2}=$ 0.64. In order to increase the efficiency we require to see only one of them, and the efficiency to detect at least one of them is then 0.96. Therefore, if there is only one Higgs boson satisfying the $\beta \gamma$ cut in the final state, the detection efficiency would be $80 \%$. While there are two Higgs bosons satisfying the $\beta \gamma$ cut in the final state, the detection efficiency for at least one of them would be $96 \%$. Thus, the overall efficiency is more than $50 \%$, which is 


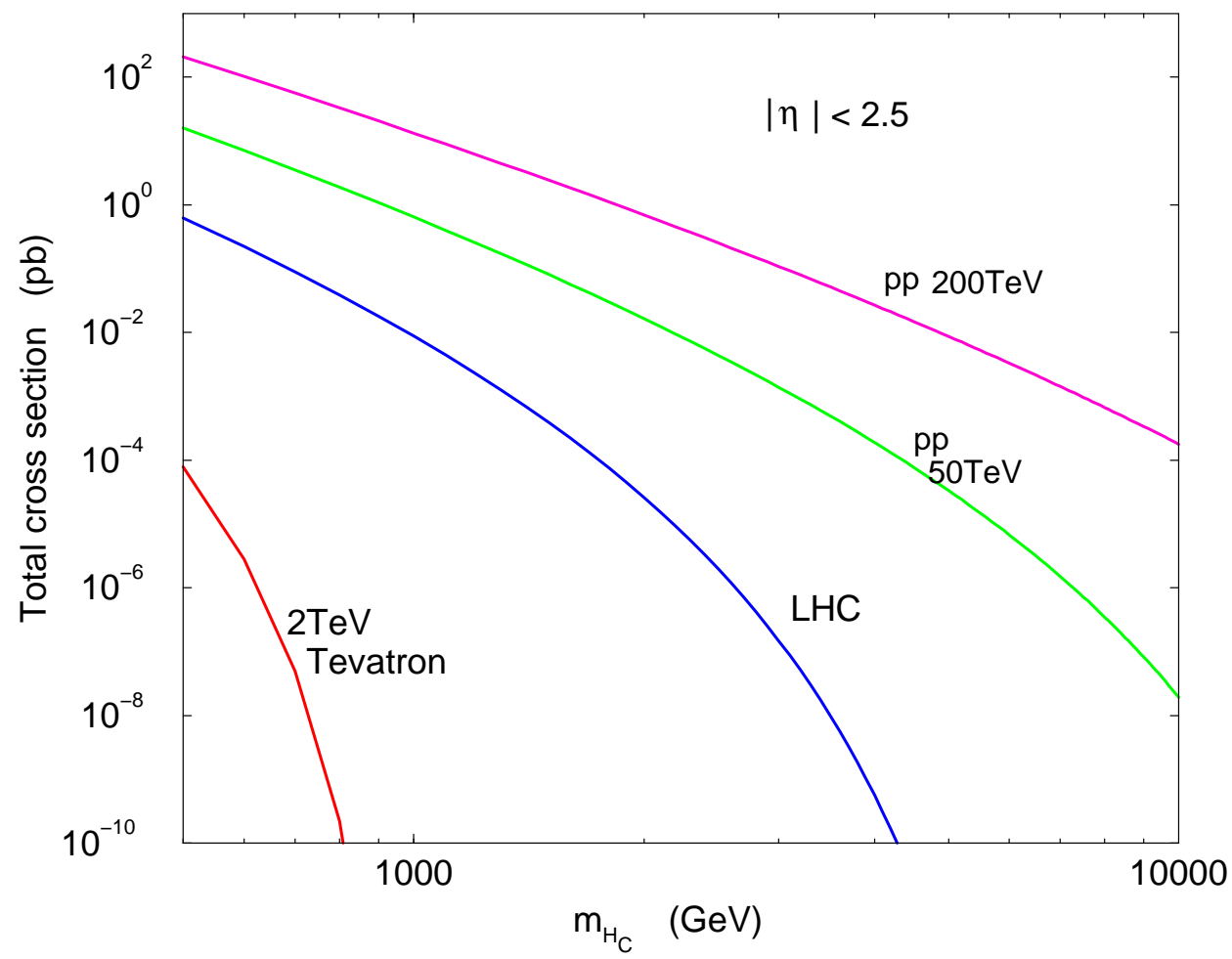

FIG. 5: Total cross sections for the production of the colored Higgs boson pair at the Tevatron, LHC, and $p p$ collisions at 50 and $200 \mathrm{TeV}$. A pseudorapidity cut $|\eta|<2.5$ is applied.

sufficient for a sizable cross section. We show the final cross sections for various energies and mass $m_{H_{C}}$ with the selection cuts applied and efficiencies in Table \. The number of observed events shown in Table \includes the following factors:

(i) a probability of $1 / 2$ that a colored Higgs boson will hadronize into an electrically charged particle;

(ii) requiring at least one of the colored Higgs bosons satisfying the selection cuts on $\eta$ of Eq. (77) and on $\beta \gamma$ of Eq. (6);

(iii) an efficiency factor of 0.8 for each detected track (requiring to detect at least one track), and

(iv) both channels of $H_{C} H_{C}^{*}$ and $\bar{H}_{C} \bar{H}_{C}^{*}$ production.

Since the search is background free, the discovery or evidence of existence for the colored Higgs bosons depends crucially on the number of observed events, which we choose to be 10 events. The run II with an integrated luminosity of $20 \mathrm{fb}^{-1}$ at the Tevatron is sensitive 


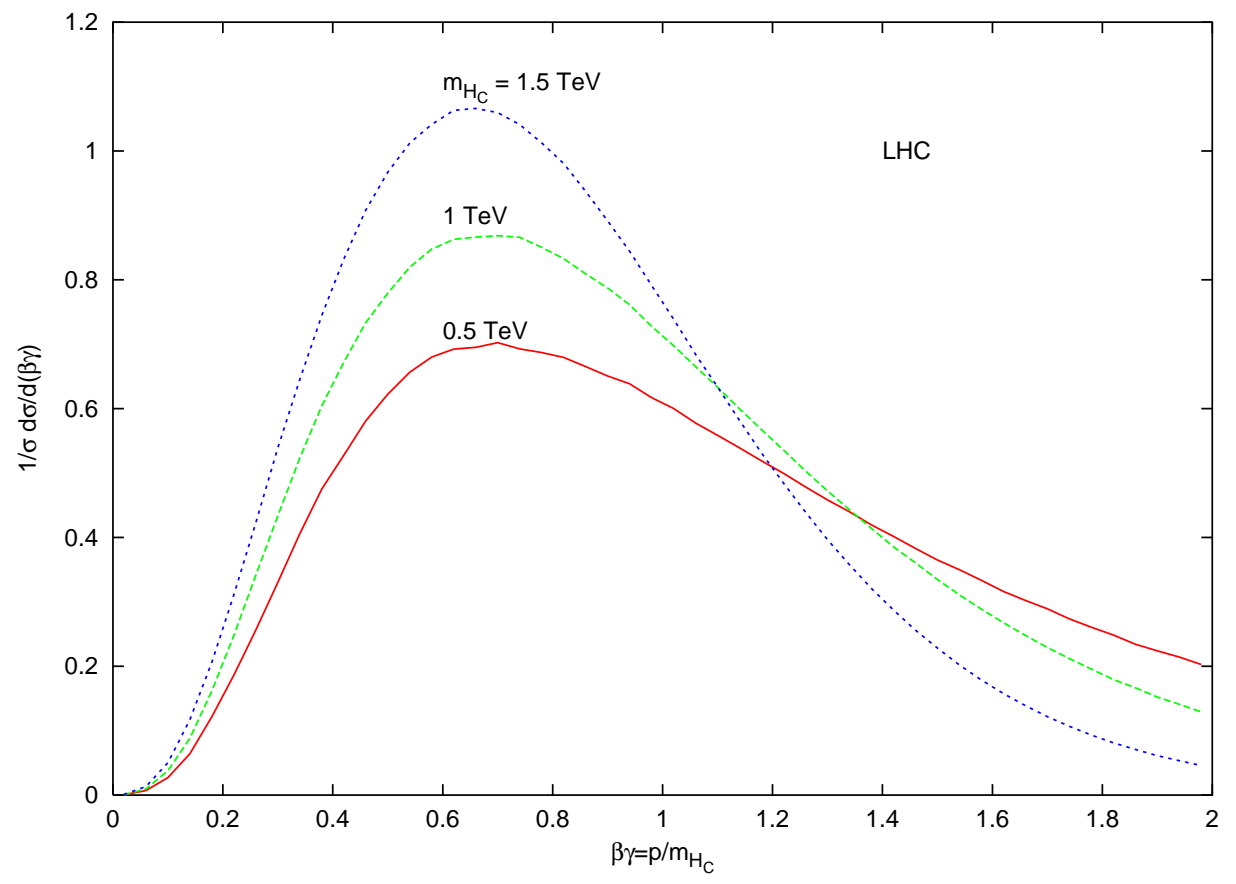

FIG. 6: Normalized differential cross section $1 / \sigma d \sigma / d(\beta \gamma)$ vs $\beta \gamma=p / m_{H_{C}}$ for $m_{H_{C}}=0.5,1,1.5$ $\mathrm{TeV}$ at the LHC.

up to about $400 \mathrm{GeV}$ while the LHC with an integrated luminosity of $100 \mathrm{fb}^{-1}$ can probe up to about $1.5 \mathrm{TeV}$. The VLHC running at $50 \mathrm{TeV}$ and $200 \mathrm{TeV}$ is sensitive to a colored Higgs boson of mass up to 3.5 and $9 \mathrm{TeV}$, respectively.

\section{SUMMARY}

The presence of light color-triplet Higgs fields in TeV mass scale is an alternative signature for GUT, instead of proton decay. This is made possible through some mechanisms to suppress the Yukawa couplings of the triplets to the matter fermions. We have reviewed three possible models by Dvali [3], by Haba and Maru [4], and by Goldberger, Nomura and Smith [5]. The former used a discrete symmetry while the latter two used a setup in extra dimensions to achieve the suppression.

The novel signature of these TeV colored Higgs bosons would be like massive stable charged particles, "heavy muons", producing a track in the central tracking chamber and penetrating to the outer muon system. Such a signature is background free and the momentum can be measured, which enables determination of the mass of the particle via the 
ionization-energy loss spectrum. We have demonstrated in details that we employed a reasonably conservative requirement on $\beta \gamma=p / M$ cut on the massive charged particle such that it can penetrate to the outer muon system and produce a charged track in the central tracking chamber.

We have calculated the production cross sections and the number of signal events of the colored Higgs bosons. We have taken into account the fact that only half of the colored Higgs bosons would hadronize into charged particles and an efficiency of $80 \%$ to detect a track. The number of observable events is increased by relaxing the requirement to seeing both colored Higgs bosons to seeing at least one. The Tevatron Run IIb may be able to discover a colored Higgs boson up to about $400 \mathrm{GeV}$ if an order of 10 events is required for discovery. The LHC with an accumulated luminosity of $100 \mathrm{fb}^{-1}$ is sensitive to about 1.5 TeV. The VLHC running at 50 and $200 \mathrm{TeV}$ is sensitive up to 3.5 and $9 \mathrm{TeV}$, respectively.

A few more comments are in order.

(i) In this work, we have assumed that the colored Higgs bosons are stable. An alternative is that the colored Higgs boson decays into a gluino and a colored higgsino if kinematically allowed. The colored higgsino is the supersymmetric partner of the colored Higgs boson. This decay would also be phenomenologically interesting because the colored higgsino would be likely to hadronize into a massive stable charged particle because there are no other lighter particles that it can decay to, and the gluino would decay into quarks and squarks producing jets and missing energies.

(ii) Since the colored Higgs bosons are stable over cosmological time scale, they have a relic density since the early universe. Our preliminary estimate of the relic density of $H_{C}$ is of order of $\Omega_{H_{C}} h^{2} \sim 0.01-0.05$ for $m_{H_{C}}=1-2 \mathrm{TeV}$ [19], which is an interesting fraction of the observed cold dark matter density $\Omega_{\mathrm{CDM}} h^{2}=0.12 \pm 0.04$ at $95 \%$ C.L. [20].

\section{Acknowledgments}

G.C.C. thanks NCTS for warm hospitality during his visit. He is also grateful to N. Haba for discussion. The work of G.C.C. is supported in part by the Grant-in-Aid for Science Research, Ministry of Education, Science and Culture, Japan (No.13740149). K.C. 
is supported the National Center for Theoretical Sciences under a grant from the National Science Council of Taiwan R.O.C.

[1] S. Dimopoulos and H. Georgi, Nucl. Phys. B 193, 150 (1981);

N. Sakai, Z. Phys. C 11, 153 (1981).

[2] For example, see;

A. Masiero, D. V. Nanopoulos, K. Tamvakis and T. Yanagida, Phys. Lett. B 115, 380 (1982);

I. Antoniadis, J. R. Ellis, J. S. Hagelin and D. V. Nanopoulos, Phys. Lett. B 194, 231 (1987);

S. Dimopoulos and F. Wilczek, NSF-ITP-82-07;

K. Inoue, A. Kakuto and H. Takano, Prog. Theor. Phys. 75, 664 (1986);

Y. Kawamura, Prog. Theor. Phys. 105, 999 (2001).

[3] G. R. Dvali, Phys. Lett. B 372, 113 (1996).

[4] N. Haba and N. Maru, Phys. Lett. B 532, 93 (2002).

[5] W. Goldberger, Y. Nomura, and D. Smith, hep-ph/0209158.

[6] L. Randall and R. Sundrum, Phys. Rev. Lett. 83, 3370 (1999).

[7] M. E. Peskin and T. Takeuchi, Phys. Rev. Lett. 65, 964 (1990), Phys. Rev. D 46, 381 (1992).

[8] G. C. Cho and K. Hagiwara, Nucl. Phys. B 574, 623 (2000).

[9] K. Hagawara et al, Particle Data Group, Phys. Rev. D66, 010001 (2002).

[10] CDF Coll., Phys. Rev. D46, R1889 (1992).

[11] A. Connolly for CDF Coll., hep-ex/9904010.

K. Hoffman for CDF Coll., hep-ex/9712032.

[12] CDF Collaboration (D. Acosta et al.), hep-ex/0211064.

[13] H. Baer, K. Cheung and J. F. Gunion, Phys. Rev. D 59, 075002 (1999)

[14] J. L. Feng and T. Moroi, Phys. Rev. D 58, 035001 (1998).

[15] W. Beenakker, R. Hopker, M. Spira and P. M. Zerwas, Nucl. Phys. B 492, 51 (1997).

[16] CTEQ Collaboration, H.L. Lai et al.. Eur. Phys. J. C12, 375 (2000).

[17] "M4 Working Group/Hadron Colliders", plenary talk given by M. Syphers at the Snowmass 2001, available online at http://www.vlhc.org/M4finalPlenary.pdf.

[18] Snowmass 2001 "The future of particle physics" meeting, Snowmass, Summer 2001.

[19] K. Cheung and G.C. Cho, in preparation. 
[20] A. Melchiorri and J. Silk, Phys. Rev. D 66, 041301 (2002). 
TABLE I: The number of signal events of massive stable charged particles due to the pair production of colored Higgs bosons $H_{C} H_{C}^{*}$ and $\bar{H}_{C} \bar{H}_{C}^{*}$ at the Tevatron, the LHC, and the VLHC of 50 and $200 \mathrm{TeV}$. We have already taken into account (i) a probability of $1 / 2$ that a colored Higgs boson will hadronize into an electrically charged particle, (ii) at least one of the colored Higgs bosons satisfying the selection cuts on $\eta$ and $\beta \gamma$, and (iii) an efficiency factor of 0.8 for each detected track.

\begin{tabular}{|c|c|c|c|c|}
\hline$m_{H_{C}}(\mathrm{TeV})$ & $\begin{array}{c}\text { Tevatron } \\
\left(\mathcal{L}=20 \mathrm{fb}^{-1}\right)\end{array}$ & $\begin{array}{c}\text { LHC } \\
\left(\mathcal{L}=100 \mathrm{fb}^{-1}\right)\end{array}$ & $\begin{array}{l}\text { VLHC } 50 \mathrm{TeV} \\
\left(\mathcal{L}=100 \mathrm{fb}^{-1}\right)\end{array}$ & $\begin{array}{l}\text { VLHC } 200 \mathrm{TeV} \\
\left(\mathcal{L}=100 \mathrm{fb}^{-1}\right)\end{array}$ \\
\hline 0.2 & 1900 & $1.5 \times 10^{6}$ & $1.5 \times 10^{7}$ & $1.2 \times 10^{8}$ \\
\hline 0.3 & 160 & $2.3 \times 10^{5}$ & $3.1 \times 10^{6}$ & $2.8 \times 10^{7}$ \\
\hline 0.4 & 14 & $5.5 \times 10^{4}$ & $9.6 \times 10^{5}$ & $9.7 \times 10^{6}$ \\
\hline 0.5 & 0.9 & $1.7 \times 10^{4}$ & $3.7 \times 10^{5}$ & $4.3 \times 10^{6}$ \\
\hline 0.6 & - & 6400 & $1.7 \times 10^{5}$ & $2.1 \times 10^{6}$ \\
\hline 0.8 & - & 1200 & $4.7 \times 10^{4}$ & $7.1 \times 10^{5}$ \\
\hline 1.0 & - & 285 & $1.6 \times 10^{4}$ & $2.9 \times 10^{5}$ \\
\hline 1.2 & - & 81 & 6800 & $1.4 \times 10^{5}$ \\
\hline 1.4 & - & 26 & 3100 & $7.4 \times 10^{4}$ \\
\hline 1.5 & - & 15 & 2200 & $5.6 \times 10^{4}$ \\
\hline 1.6 & - & 8.8 & 1600 & $4.3 \times 10^{4}$ \\
\hline 1.8 & - & 3.1 & 830 & $2.6 \times 10^{4}$ \\
\hline 2.0 & - & 1.2 & 470 & $1.6 \times 10^{4}$ \\
\hline 2.5 & - & - & 130 & 6100 \\
\hline 3.0 & - & - & 43 & 2700 \\
\hline 3.5 & - & - & 15 & 1300 \\
\hline 4.0 & - & - & 6.4 & 690 \\
\hline 5.0 & - & - & 1.2 & 230 \\
\hline 6.0 & - & - & - & 90 \\
\hline 7.0 & - & - & - & 40 \\
\hline 8.0 & - & - & - & 19 \\
\hline 9.0 & - & - & - & 9.7 \\
\hline 10.0 & - & - & - & 5.2 \\
\hline
\end{tabular}

